\title{
PHOTON DOMINATED REGIONS: OBSERVATIONS OF [C I]
} AND CO

\author{
JOCELYN KEENE, D. C. LIS AND T. G. PHILLIPS \\ California Institute of Technology, 320-47 \\ Pasadena, CA 91125, USA \\ AND \\ P. SCHILKE \\ Universität zu Köln, I. Physikalisches Institut \\ Zülpicher Str. 77, D-50937, Köln, Germany
}

\begin{abstract}
Since the construction of the first specialized submillimeter telescopes nearly a decade ago, there has been extensive mapping of astronomical sources in the previously difficult to observe ground-state fine-structure transition of [C I] at $492 \mathrm{GHz}$. We discuss recent CSO observations of [C I] and ${ }^{13} \mathrm{CO}(2 \rightarrow 1)$ lines toward bright photon-dominated regions (PDRs). These observations have revealed an interesting effect. In many sources and over a broad range of column densities, the observed intensity of the [CI] line is proportional to that of the ${ }^{13} \mathrm{CO}(2 \rightarrow 1)$ line with exceptions mainly in dense, active star-forming regions, where C I appears to be under-abundant relative to ${ }^{13} \mathrm{CO}$. In this paper we quantitatively display this effect.
\end{abstract}

\section{Introduction}

This review is intended to discuss observations of Photon Dominated Regions (PDRs) and as such will not discuss the theory or calculations to any great extent. That task is left to the following review by Dalgarno. Nevertheless, because this is the first paper on the subject, we will present a short introduction to the topic.

The earliest reference that we can find to the chemical structure of a PDR is by Langer (1976) who calculated the structure of a cold interstellar cloud illuminated by the ambient interstellar radiation field. A more recent 
series of calculations have been performed by Tielens \& Hollenbach (1985) and their collaborators in subsequent papers starting with the calculation of the structure of a PDR near a luminous $\mathrm{O}$ star, such as the Orion nebula. Calculations have also been made by van Dishoeck \& Black (1988), Sternberg \& Dalgarno (1995), Le Bourlot et al. (1993) and their collaborators in subsequent papers.

\section{Simple PDR models}

There are three important aspects of the carbon atom that influence its distribution in the interstellar medium:

- Carbon is the fourth most abundant element in the universe with an abundance relative to hydrogen in the local ISM thought to be about $2.4 \times 10^{-4}$ (Cardelli et al. 1996). Its abundance is about $1 / 2$ that of oxygen.

- Its ionization threshold, $11.26 \mathrm{eV}$, is the lowest of the six most abundant elements. This means that, among atoms, carbon has little competition for interstellar ultraviolet radiation with photon energy between 11.26 and $13.6 \mathrm{eV}$ and that it will be easily ionized unless it is shielded by dust. Therefore, in low-density, unshielded regions, carbon should mostly be found in its singly ionized form (C II).

- The dissociation threshold of the most common molecular form of carbon, the carbon monoxide molecule, $11.09 \mathrm{eV}$, is very close to the ionization threshold of the carbon atom. Carbon monoxide is a very stable molecule and is the endpoint of chemical reactions in a shielded environment. Therefore in a shielded environment it would be reasonable to expect most of the gas-phase carbon to be in the form of $\mathrm{CO}$.

The implication of these basic facts is that CI should form a fairly narrow layer between $\mathrm{CII}$ on the outside and $\mathrm{CO}$ within a molecular cloud. The result of a typical model calculation (Tielens \& Hollenbach 1985; van Dishoeck \& Black 1988) which has a geometry of a dense, plane-parallel cloud illuminated by an ultraviolet field, is that the CI forms a layer partially coinciding with the outside of the $\mathrm{CO}$ layer, but having a depth of only about $5-7 \mathrm{mag}$ of visual extinction. The total column density of this layer of $\mathrm{CI}$ is about $5 \times 10^{17} \mathrm{~cm}^{-2}$.

It turns out that the column density of $\mathrm{CI}$ created by such a straightforward model is relatively insensitive to most parameters. It is not very sensitive to either the radiation field or the density. Moreover, the excitation of the ${ }^{3} P_{1}$ level of $\mathrm{CI}$ is insensitive to both temperature (for $T>15 \mathrm{~K}$ ) and density (for $n>10^{3} \mathrm{~cm}^{-3}$ ). As a result, the integrated intensity of the ${ }^{3} P_{1} \rightarrow{ }^{3} P_{0}$ line of [C I] in PDRs should be about $40 \mathrm{~K} \mathrm{~km} \mathrm{~s}^{-1}$ under many conditions. Of course this brightness may be decreased in regions with low 
temperature (i.e., a dark cloud), low column density (i.e., a diffuse cloud), or high opacity (i.e., a small linewidth). It may be increased in a tilted PDR or by superposition of several PDRs.

Plane-parallel PDR models would have no reason to have [CI] emission proportional to an optically thin tracer of the molecular material, such as ${ }^{13} \mathrm{CO}$, since their distribution within the cloud is not predicted to be the same. Instead, as the molecular column density increases from zero, we would expect a concurrent increase in the [CI] intensity followed by saturation of the [CI] intensity at $40 \mathrm{~K} \mathrm{~km} \mathrm{~s}^{-1}$ (or higher, in the case of a tilted PDR), beyond which we observe molecular material within the cloud which has little or no coexisting atomic carbon. Mysteriously, however, in many objects a proportionality does exist between ${ }^{13} \mathrm{CO}$ and [CI]. Moreover, we observe intensities up to $200 \mathrm{~K} \mathrm{~km} \mathrm{~s}^{-1}$ (column densities up to $N(\mathrm{C} \mathrm{I})=2.5 \times 10^{18} \mathrm{~cm}^{-2}$ ) near the M17 H II region, and even higher in a single spectral feature near the Galactic Center (the $50 \mathrm{~km} \mathrm{~s}^{-1}$ cloud). These observations lead us to doubt the appropriateness of simple PDR models and may act as a test of more complex models.

Since the publication of the Tielens \& Hollenbach (1985) model, many more PDR models have been published, some with interesting departures from the model discussed above. For example, the chemistry in the model by Le Bourlot et al. (1993) is different from that in many others. It shows high abundances of $\mathrm{CI}$ for regions of high ionization fraction, and low abundances where the ionization fraction is low. Clumpy models have been explored by Meixner \& Tielens $(1993,1995)$ and Spaans (1996). Both these variations on simple PDR models can spread the abundance of CI physically deeper into the cloud and thus produce a proportionality between $\mathrm{CI}$ and ${ }^{13} \mathrm{CO}$. The PDR model of Le Bourlot et al. (1993) can generate large amounts of $\mathrm{CI}$ if the ionization fraction is high throughout a thick cloud. In clumpy models the total amount of $\mathrm{CI}$ is approximately the same as in simpler models, but, possibly in future models, there could be added complexities such as multiple, spatially-distributed sources of dissociating radiation to increase the total amount of $\mathrm{CI}$.

\section{PDR maps of [C I] and ${ }^{13} \mathrm{CO}$}

How do the simple PDR theories compare with observations? In early strip scans of [C I] emission by Keene et al. (1985) and Genzel et al. (1988) it was shown that a high abundance of CI extends quite far into molecular clouds from the PDRs, much further than could be accounted for by simple planeparallel models of PDRs. This was interpreted by Stutzki et al. (1988) as evidence for clumpy structure of molecular clouds allowing deep penetration of UV radiation into clouds, effectively producing small PDRs at the surface 


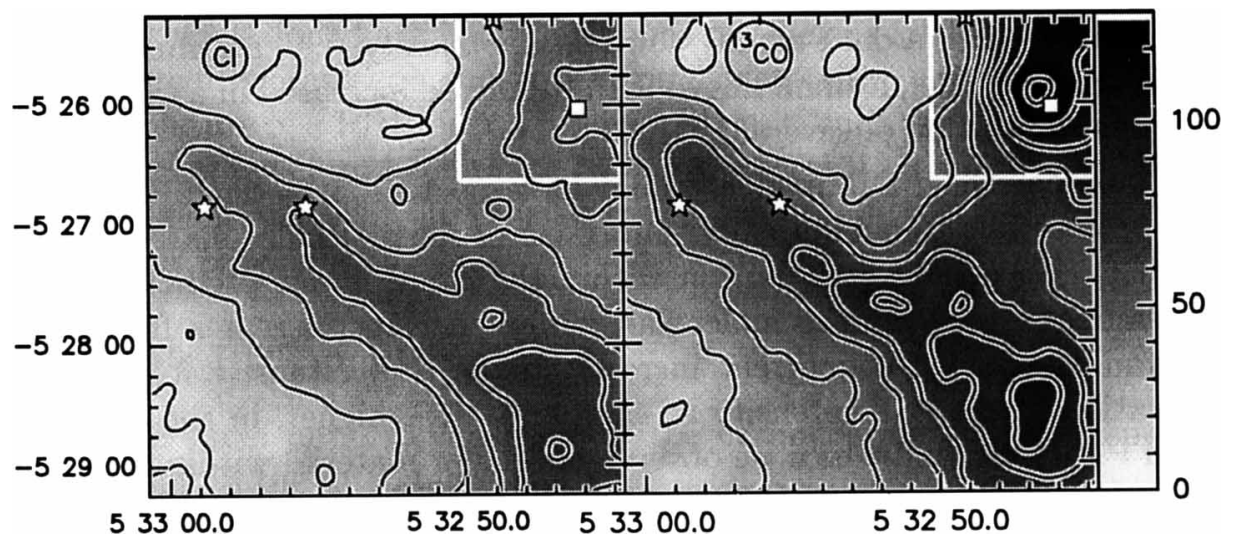

Figure 1. [C I] (left) and ${ }^{13} \mathrm{CO}(2 \rightarrow 1)$ (right) maps of the Orion Bar Region (Tauber et al. 1995). The open square marks the position of Orion South; the stars mark $\theta^{1}$ Ori $\mathrm{C}$ (extreme top) and $\theta^{2}$ Ori A \& B. The white border encloses the area used for Orion South in Figure 2. The greyscales are the same for the two figures, the contours are $12 \%$ of the peak flux of ${ }^{13} \mathrm{CO}$, or $15.6 \mathrm{~K} \mathrm{~km} \mathrm{~s}^{-1}$, integrated between 3 and $15 \mathrm{~km} \mathrm{~s}^{-1}$. The circle at the top of each map indicates the effective beamsize, $22^{\prime \prime}$ for [C I] and $33^{\prime \prime}$ for ${ }^{13} \mathrm{CO}$.

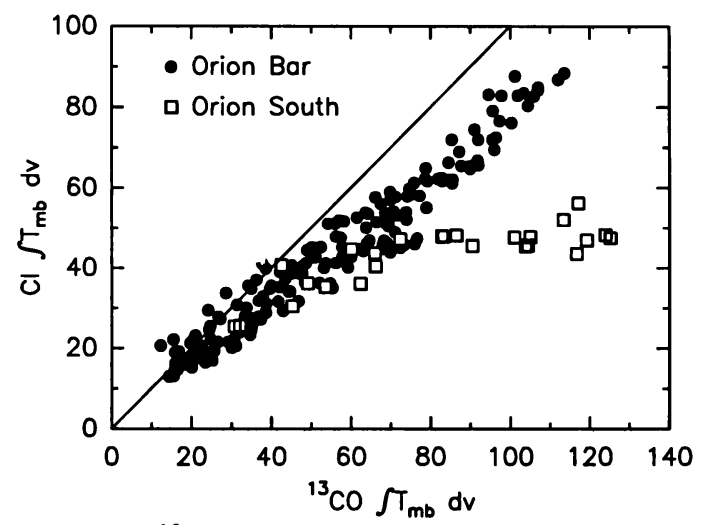

Figure 2. Correlation of the ${ }^{13} \mathrm{CO}$ and [C I] maps of the Orion Bar region. The [C I] intensities have been smoothed to the $33^{\prime \prime}$ resolution of the ${ }^{13} \mathrm{CO}$ map. The straight line is for reference only and has a slope of unity. In this figure the points for Orion South (open squares) display the behavior that might be expected for a simple plane-parallel PDR. The points for the Orion Bar do not.

of each illuminated clump.

Since these early observations were made, there have been many improvements in the instruments used for observing the [C I] lines. The early observations suffered from being made with receivers with small bandwidth or low sensitivity and with small telescopes. Now they are observable with higher-sensitivity, broad-band receivers on submillimeter telescopes ranging from large (JCMT, CSO, SMT), to medium (KOSMA), and on to highly 
specialized small telescopes (AST/RO and soon SWAS). These improvements have allowed mapping of reasonably large areas ( $10 \mathrm{sq}$ arcmin) with high angular resolution in the $492 \mathrm{GHz}{ }^{3} P_{1} \rightarrow{ }^{3} P_{0}$ line of [CI] in a few hours time. The $809 \mathrm{GHz}{ }^{3} P_{2} \rightarrow{ }^{3} P_{1}$ line is just now becoming available with the advent of new high-frequency SIS receivers. Very little work has so far been done in this line, though see the poster by Graf et al. in the IAU 178 Abstract book. As a result we can now begin to make much more detailed comparisons between the distributions of [C I] and molecular species and compare these distributions with PDR theories.

One of the earliest high-quality broad-band [CI] maps was the map of the Orion Bar made at the CSO by Tauber et al. (1995). It has been long noted (Keene 1990) that the $492 \mathrm{GHz}$ line of [C I] typically resembles the lines of ${ }^{13} \mathrm{CO}(1 \rightarrow 0)$ and $(2 \rightarrow 1)$ in intensity, lineshape, and spatial distribution. Accordingly, we compared our map of [CI] in the Orion Bar to a map of ${ }^{13} \mathrm{CO}(2 \rightarrow 1)$. The two maps are shown in Figure 1 . Here the [C I] map, which has a beamsize of $\sim 15^{\prime \prime}$ and pixel separation of $7.5^{\prime \prime}$ has been smoothed to $22^{\prime \prime}$ resolution. The ${ }^{13} \mathrm{CO}$ map has $33^{\prime \prime}$ resolution and $15^{\prime \prime}$ pixel separation. To compare the two species, pixel by pixel, we smoothed the [CI] map to the resolution of the ${ }^{13} \mathrm{CO}$ map. The comparison of the resulting $15^{\prime \prime}$ pixels is shown in Figure 2.

We were very interested to find that there is an almost exact correspondence of the $\left[\mathrm{CI}\right.$ ] map to the ${ }^{13} \mathrm{CO}(2 \rightarrow 1)$ map, giving the unexpected correlation between [C I] and ${ }^{13} \mathrm{CO}$ mentioned in the previous section. The single exception to this lies in the Orion South region (Keene et al. 1982) enclosed by the white border in Figure 1, which is bright in ${ }^{13} \mathrm{CO}$ but not at all in [C I]. This is the densest region in this map, having densities of $>10^{5}-10^{6} \mathrm{~cm}^{-3}$ as shown by measurements of $\mathrm{NH}_{3}$ and $\mathrm{HC}_{3} \mathrm{~N}$ (Batrla et al. 1983; Martín-Pintado et al. 1990), and has internal star formation, as revealed by the presence of a molecular outflow (Schmid-Burgk et al. 1989). In this very dense region, the [CI] intensity $\left(40 \mathrm{~K} \mathrm{~km} \mathrm{~s}^{-1}\right)$ and the shape of the $[\mathrm{CI}]-{ }^{13} \mathrm{CO}$ correlation looks like those that might be expected for a simple PDR as discussed in the previous section.

Inspired by this beautiful correspondence of [C I] with ${ }^{13} \mathrm{CO}$ we set out to map other bright PDRs in the same lines at the CSO. [C I] and ${ }^{13} \mathrm{CO}$ maps of the central part of the Orion Molecular Cloud are shown in Figure 3. This region has also been mapped previously by Büttgenbach (1993; shown by Keene 1995), White \& Padman (1991) and White \& Sandell (1995). The same general features are visible in all these maps. These maps demonstrate that not all PDRs show such a tight relationship between [C I] and ${ }^{13} \mathrm{CO}$. In fact, in the OMC-1 region the correspondence is rather poor. The ${ }^{13} \mathrm{CO}$ peaks near IRc2, a region with active star formation and high densities, but [CI] avoids this region. At the bottom of this map we again 


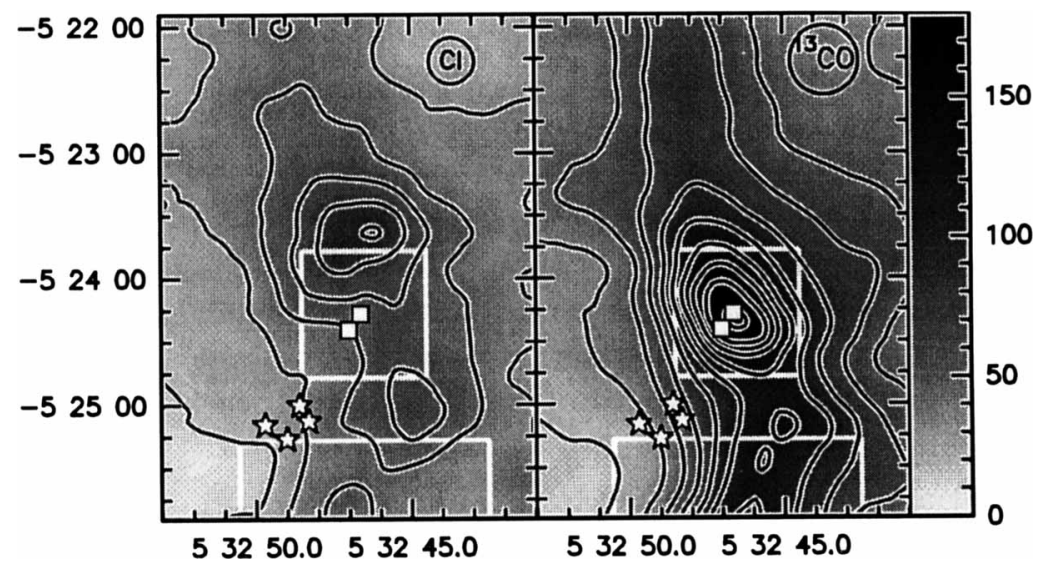

Figure 3. [C I] (left) and ${ }^{13} \mathrm{CO}(2 \rightarrow 1)$ (right) maps of the center of OMC-1. The small squares mark the positions of BN and Radio Source 1 (Menten \& Reid 1995); the stars mark the Trapezium. The white borders enclose the regions used for the Orion Outflow and Orion South in Figure 4. The greyscales are the same for the two figures, the contours are $10 \%$ of the peak flux of ${ }^{13} \mathrm{CO}$, or $18 \mathrm{~K} \mathrm{~km} \mathrm{~s}^{-1}$, integrated between 4 and $14 \mathrm{~km} \mathrm{~s}^{-1}$.

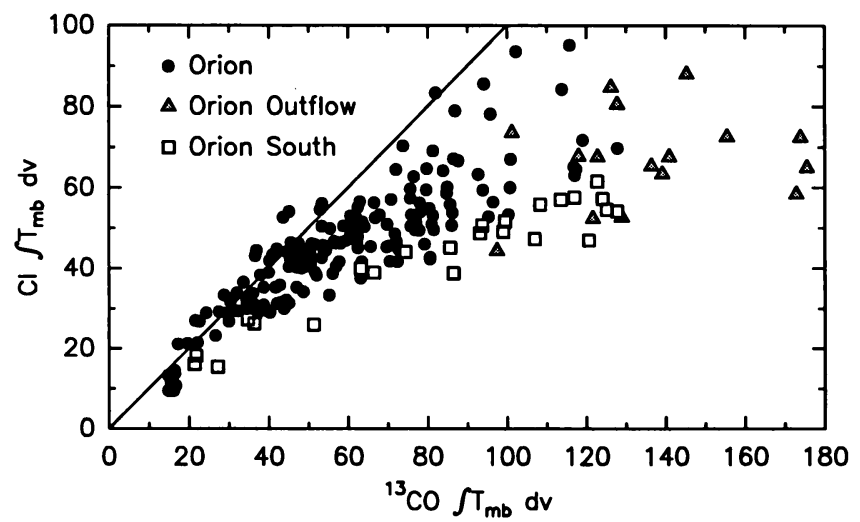

Figure 4. Correlation of the ${ }^{13} \mathrm{CO}$ and [C I] maps of the OMC-1 region. The [C I] intensities have been smoothed to the $33^{\prime \prime}$ resolution of the ${ }^{13} \mathrm{CO}$ map.

see that the [CI] avoids the Orion South region, as shown in Figure 1.

The [C I] to ${ }^{13} \mathrm{CO}$ pixel-pixel correlation is shown in Figure 4. Here, as in the maps, the correspondence is not striking. In fact there is a large dispersion in the relationship at [C I] intensity values $>30-40 \mathrm{~K} \mathrm{~km} \mathrm{~s}^{-1}$. The shaded coding of the dots in this figure gives a clue to why the correlation is so poor. The white squares show the region of Orion South, which was already seen in Figure 2 to display a non-linear relationship between [C I] and ${ }^{13} \mathrm{CO}$ emission. The gray triangles show the correlation for the region of the OMC-1 outflow near IRc2. Here the widths of the ${ }^{13} \mathrm{CO}$ lines are large but those of [CI] are not. This implies that the abundance of CI in 


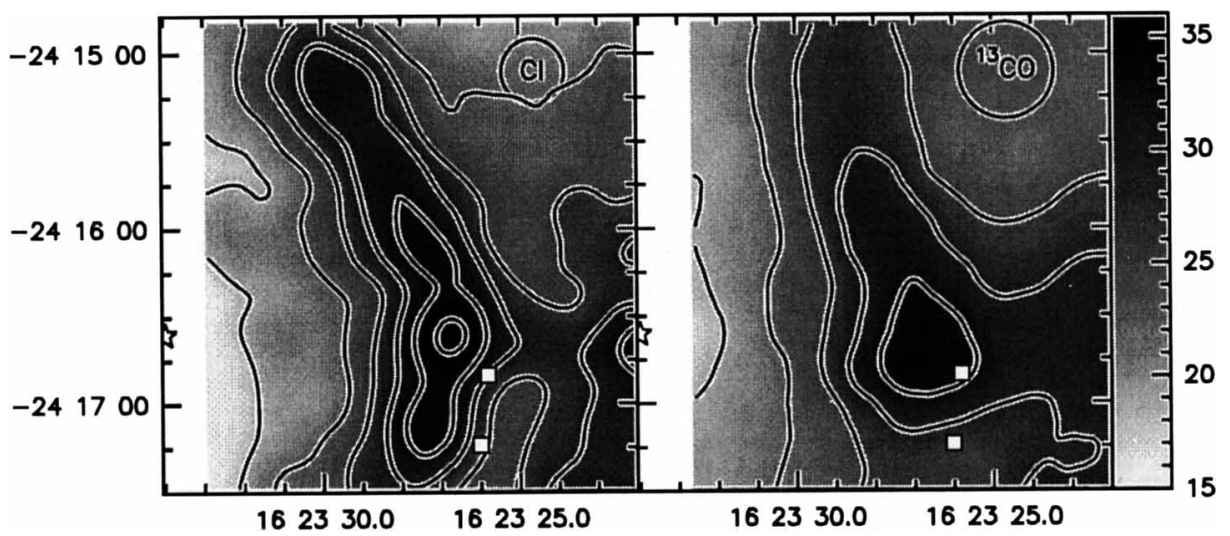

Figure 5. [C I] (left) and ${ }^{13} \mathrm{CO}(2 \rightarrow 1)$ (right) maps of the Oph A region. The squares mark the positions of SM 1 and SM $1 \mathrm{~N}$; the star at the extreme left marks the position of S1, also known as BZ4. The greyscales are the same for the two figures, the contours are $10 \%$ of the peak flux of [C I], or $3.6 \mathrm{~K} \mathrm{~km} \mathrm{~s}^{-1}$, integrated between 2 and $5 \mathrm{~km} \mathrm{~s}^{-1}$.

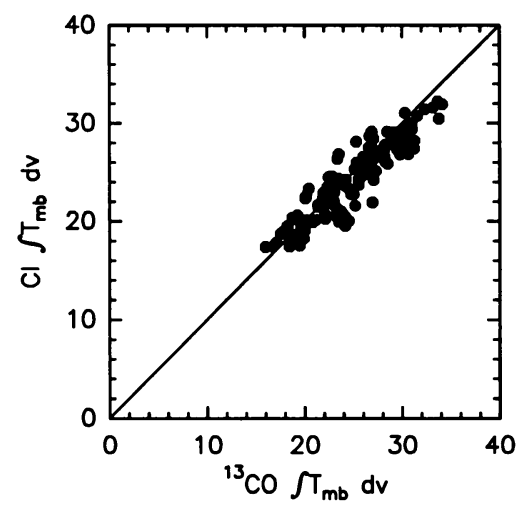

Figure 6. Correlation of the ${ }^{13} \mathrm{CO}$ and [C I] maps of the Oph A region. The [C I] intensities have been smoothed to the $33^{\prime \prime}$ resolution of the ${ }^{13} \mathrm{CO}$ map.

the outflowing gas is considerably lower than its abundance in the general molecular cloud. However, it is not merely the widths of the [CI] lines near IRc2 which are small compared to ${ }^{13} \mathrm{CO}$ but also their peak intensities. Apparently, CI is also under-abundant in the more quiescent gas in the IRc2 vicinity.

Other observations of PDR regions include the Oph A region. The rim of emission in Oph A seen in Figure 5, is caused by S1 (Grasdalen et al. 1973), an embedded $\sim \mathrm{B} 3$ star which produces the compact H II region BZ4 (Brown \& Zuckerman 1975). Immediately west of this rim of emission lie the submillimeter sources SM1, SM1 N, and SM2 (Ward-Thompson et al. 1989, André et al. 1993). East of the [CI] arc, closer to the star S1, is an 


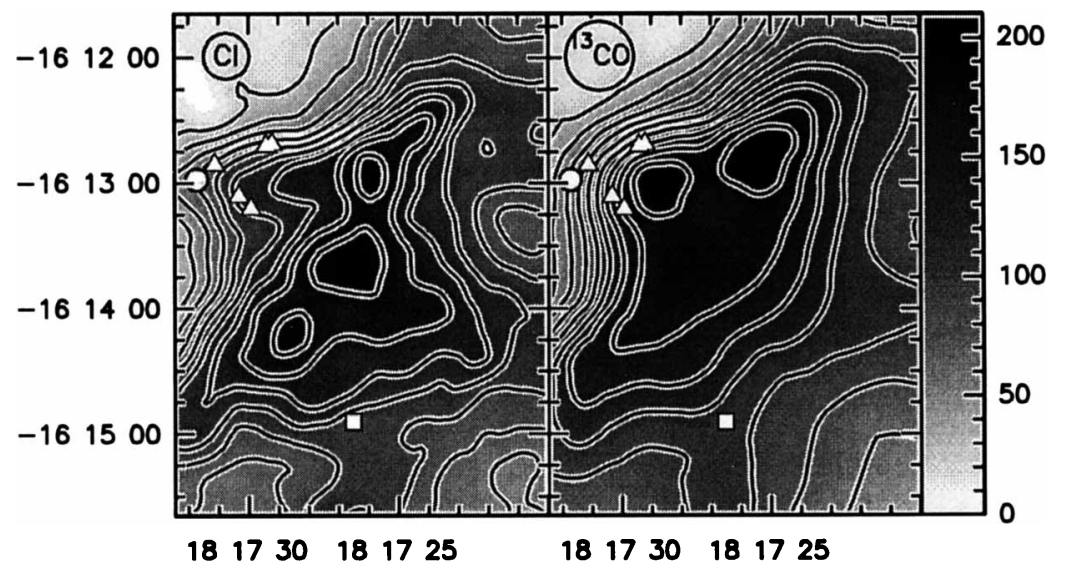

Figure 7. [C I] (left) and ${ }^{13} \mathrm{CO}(2 \rightarrow 1)$ (right) maps of M17. The square marks the position of the Kleinmann-Wright source; the triangles mark the positions of some $\mathrm{H}_{2} \mathrm{O}$ masers (Jaffe et al. 1981, Massi et al. 1988); the small circle marks the position of an ultracompact $\mathrm{H}$ II region (Felli et al. 1984). The greyscales are the same for the two figures, the contours are $10 \%$ of the peak flux, or $21 \mathrm{~K} \mathrm{~km} \mathrm{~s}^{-1}$, integrated between 10 and $30 \mathrm{~km} \mathrm{~s}^{-1}$.

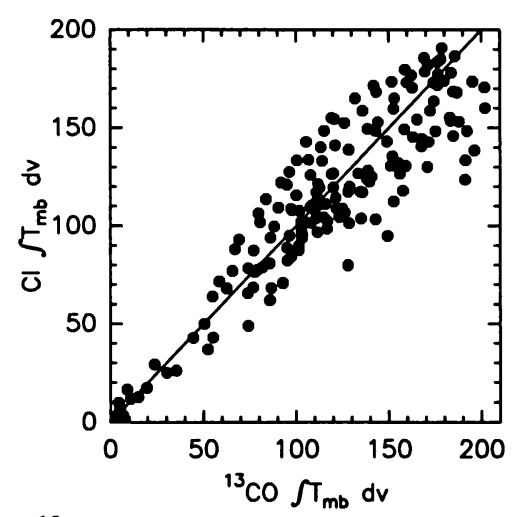

Figure 8. Correlation of the ${ }^{13} \mathrm{CO}$ and [C I] maps of the $\mathrm{M} 17$ region. The [C I] intensities have been smoothed to the $33^{\prime \prime}$ resolution of the ${ }^{13} \mathrm{CO}$ map.

arc of radio continuum emission delineating an ionization front ( $\mathrm{P}$. André, priv. comm.). The dynamic range of these maps is small because there is moderately bright emission all over the region. Nevertheless, the [C I] shows an extremely good correlation with ${ }^{13} \mathrm{CO}$, as can be seen in Figure 6.

Finally, maps of M17 are shown in Figure 7. Here the correlation between [C I] and ${ }^{13} \mathrm{CO}$ emission is very good at low levels but not at the highest contour levels. This behavior is shown explicitly in the pixel-pixel plot in Figure 8. Interestingly, in M17, as in Orion South and Orion IRc2, the [CI] tends to avoid the region with the most activity. The bright ${ }^{13} \mathrm{CO}$ clump at about $18^{\mathrm{h}} 17^{\mathrm{m}} 30^{\mathrm{s}},-16^{\circ} 13^{\prime} 00^{\prime \prime}(\mathrm{B} 1950)$ has been observed in $\mathrm{NH}_{3}$ 
(Massi et al. 1988), $\mathrm{C}^{18} \mathrm{O}$ (Stutzki \& Güsten 1990), and CS (Stutzki \& Güsten 1990; Wang et al. 1993). The edge of this clump shows up as a bright arc in radio continuum maps (Felli et al. 1984). Between the radio arc and the clump center are nestled some $\mathrm{H}_{2} \mathrm{O}$ masers and a compact $\mathrm{H}$ II region, denoting recent massive star-formation. The densities throughout the whole region, measured with multi-transitional CS lines (Wang et al. 1993), are on the order of $5 \times 10^{5} \mathrm{~cm}^{-3}$ but it is likely that the filling factor of dense material in this "northern condensation" is higher than is prevalent for most of the region (Stutzki \& Güsten 1990). In spite of this condensation having the most prominent associated PDR, it is noticeably weaker in [C I] than in ${ }^{13} \mathrm{CO}$.

All the data that we have presented above have been given in terms of line intensities. We note here that to make a more accurate comparison between $\mathrm{CI}$ and ${ }^{13} \mathrm{CO}$ we should convert the line intensities into column densities. Although the [C I] intensity is insensitive to variations in temperature and density for regions such as we have described here, the same is not necessarily true of the ${ }^{13} \mathrm{CO}(2 \rightarrow 1)$ intensity. It has a modest temperature dependence and may be under-excited at densities of $10^{3} \mathrm{~cm}^{-3}$. In fact, particularly in $\mathrm{M} 17$, there is evidence that the ${ }^{13} \mathrm{CO}(2 \rightarrow 1)$ line suffers from self-absorption much more than the [CI] line. This may be because of the different critical densities for the two species. The self-absorption of the ${ }^{13} \mathrm{CO}$ line may explain some of the dispersion at the high intensities in Figure 8. Because the calculation of ${ }^{13} \mathrm{CO}$ column densities requires more data than we have presented here (particularly if we wish to use an LVG or Monte-Carlo approach rather than the straightforward LTE calculation) we will leave the comparison of column densities for a future exercise.

The regions presented here have all (except the Orion Bar) been mapped at the CSO in a short space of time in early 1996. The calibration of this new data set is believed to be internally consistent. The Orion Bar data (Fig. 1; Tauber et al. 1995) have been recalibrated to match the new Orion data (Fig. 3) in their overlapping regions. We therefore believe that the slope of the relationship between [C I] and ${ }^{13} \mathrm{CO}$ is approximately correct and is significant. For all these sources presented here the slope is near unity. We have also observed sources where the slope differs considerably from unity. These sources are Sgr A (slope up to 3 in some features) and IC 443 G (slope about 4). It seems clear that the differences in slopes among these sources are too great to be caused by temperature and density effects alone. They must be related to the absolute abundance ratios of $\mathrm{CI}$ and ${ }^{13} \mathrm{CO}$, with the ratio being considerably higher in these sources. Unfortunately, these two sources have not been observed at the same time as the sources presented here so it is necessary to do further checking on the calibration of these observations. 


\section{A challenge to PDR models}

We have noted above how the intensity of the [C I ${ }^{3} P_{1} \rightarrow{ }^{3} P_{0}$ line in bright $\mathrm{PDRs}$ is often proportional to the intensity of the ${ }^{13} \mathrm{CO}(2 \rightarrow 1)$ line. Moreover, the proportionality sometimes extends to quite high intensities. Simple plane-parallel PDR models cannot explain either the proportionality or the widespread distribution of the [CI] line. More complex PDR models, for example that of Spaans (1996) which incorporates clumps into PDR theory in a physical way, can give a widespread distribution of [C I] with [C I] intensities proportional to ${ }^{13} \mathrm{CO}$. However, as of now this model has only been applied to sources with weak line (e.g., S140; Spaans \& van Dishoeck 1996). It remains to be seen if it can produce proportionality over such large areas and large ranges of intensity as is seen, for example, in M17. If not, then it may prove necessary to incorporate one of the many alternative methods of producing high $\mathrm{CI} / \mathrm{CO}$ ratios which have been discussed in the literature.

\section{Acknowledgements}

We thank the staff of the CSO for making possible the observations reported here. The CSO is supported by the NSF under contract AST93-13929.

\section{References}

André, P., Ward-Thompson, D., Barsony, M., 1993, ApJ, 406, 122

Batrla, W., Wilson, T.L., Bastien, P., Ruf, K., 1983, A\&A, 128, 279

Brown, R.L., Zuckerman, B., 1975, ApJ, 202, L125

Büttgenbach, T.H., 1993, PhD Thesis, California Institute of Technology

Cardelli, J.A., Meyer, D.M., Jura, M., Savage, B.D., 1996, ApJ, 467, 334

Felli, M., Massi, M., Churchwell, E., 1984, A\&A, 136, 53

Genzel, R., Harris, A.I., Stutzki, J., Jaffe, D.T., 1988, ApJ, 332, 1049

Grasdalen, G.L., Strom, K.M., Strom, S.E., 1973, ApJ, 184, L53

Jaffe, D.T., Wilson, T.L., Thomasson, P., 1981, ApJ, 223, L123

Keene, J., 1990, in Carbon in the Galaxy: Studies from Earth and Space, NASA CP-3061, 181

Keene, J., 1995, in the Proceedings of the 2nd Cologne-Zermatt Symposium, The Physics and Chemistry of Interstellar Molecular Clouds, eds. G. Winnewisser and G. Pelz (Berlin: Springer), 186

Keene, J., Blake, G.A., Phillips, T.G., Huggins, P.J., Beichman, C.A., 1985, ApJ, 299, 967

Keene, J., Hildebrand, R.H., Whitcomb, S.E., 1982, ApJ, 252, L11

Langer, W.D., 1976, ApJ, 206, 699

Le Bourlot, J., Pineau des Fôrets, G., Roueff, E., Flower, D.R., 1993, A\&A, 267, 337

Martín-Pintado, J., Rodríguez-Franco, A., Bachiller, R., 1990, ApJ, 357, L49

Massi, M., Felli, M., Churchwell, E., 1988, A\&A, 194, 116

Meixner, M., Tielens, A.G.G.M., 1993, ApJ, 405, 216

Meixner, M., Tielens, A.G.G.M., 1995, ApJ, 446, 907

Menten, K.M., Reid, M.J., 1995, ApJ, 445, L157

Schmid-Burgk, J. et al. 1989, A\&A, 215, 150 
Spaans, M., 1996, A\&A, 307, 271

Spaans, M., van Dishoeck, E.F., 1996, A\&A, in press

Sternberg, A., Dalgarno, A., 1995, ApJS, 99, 565

Stutzki, J., Güsten, R., 1990, ApJ, 356, 533

Stutzki, J., Stacey, G.J., Genzel, R., Harris, A.I., Jaffe, D.T., Lugten, J.B., 1988, ApJ, 332,379

Tauber, J.A., Lis, D.C., Keene, J., Schilke, P., Büttgenbach, T.H., 1995, A\&A, 297, 567

Tielens, A.G.G.M, Hollenbach, D., 1985, ApJ, 291, 722

van Dishoeck, E.F., Black, J.H., 1988, ApJ, 334, 771

Wang, Y., Jaffe, D.T., Evans, N.J.,II, Hayashi, M., Tatematsu, K., Zhou, S., 1993, ApJ, 419,707

Ward-Thompson, D., Robson, I., Whittet, D.C.B., Gordon, M.A., Walther, D.M., Duncan, W.D., 1989, MNRAS, 241, 199

White, G.J., Padman, R., 1991, Nature, 354, 511

White, G.J., Sandell, G., 1995, A\&A, 299, 179

\section{Discussion}

Wilson: Can you be sure that the [C I] line at $492 \mathrm{GHz}$ has a small optical depth? Keene: I would say, rather, that the optical depth is moderate, i.e., similar to ${ }^{13} \mathrm{CO} 1-0$ or $2-1$. This is probably one reason why the [C I] and ${ }^{13} \mathrm{CO}$ maps match so well.

Pecker: I would think that, taking into consideration the values of ionization potentials and dissociation energies of (respectively) $\mathrm{CI}, \mathrm{HI}, \mathrm{CO}, \ldots$, the radiative transfer problem has to be solved in any serious theoretical attempt to reproduce your observations of CI vs. CO. And the spectrum of the radiation field varies, thus, from point-to-point, creating a variety of situations, in a geometrically complicated region. Isn't that true?

Keene: Yes. The coupled problem of $\mathrm{C}$ photoionization, $\mathrm{CO}$ photodissociation, radiative transfer and radiation field spectrum is one that a.o. our conference organizer, Ewine, has been active in solving. The question of geometry has been attacked by Marco Spaans, who has also included these effects in his calculations. 


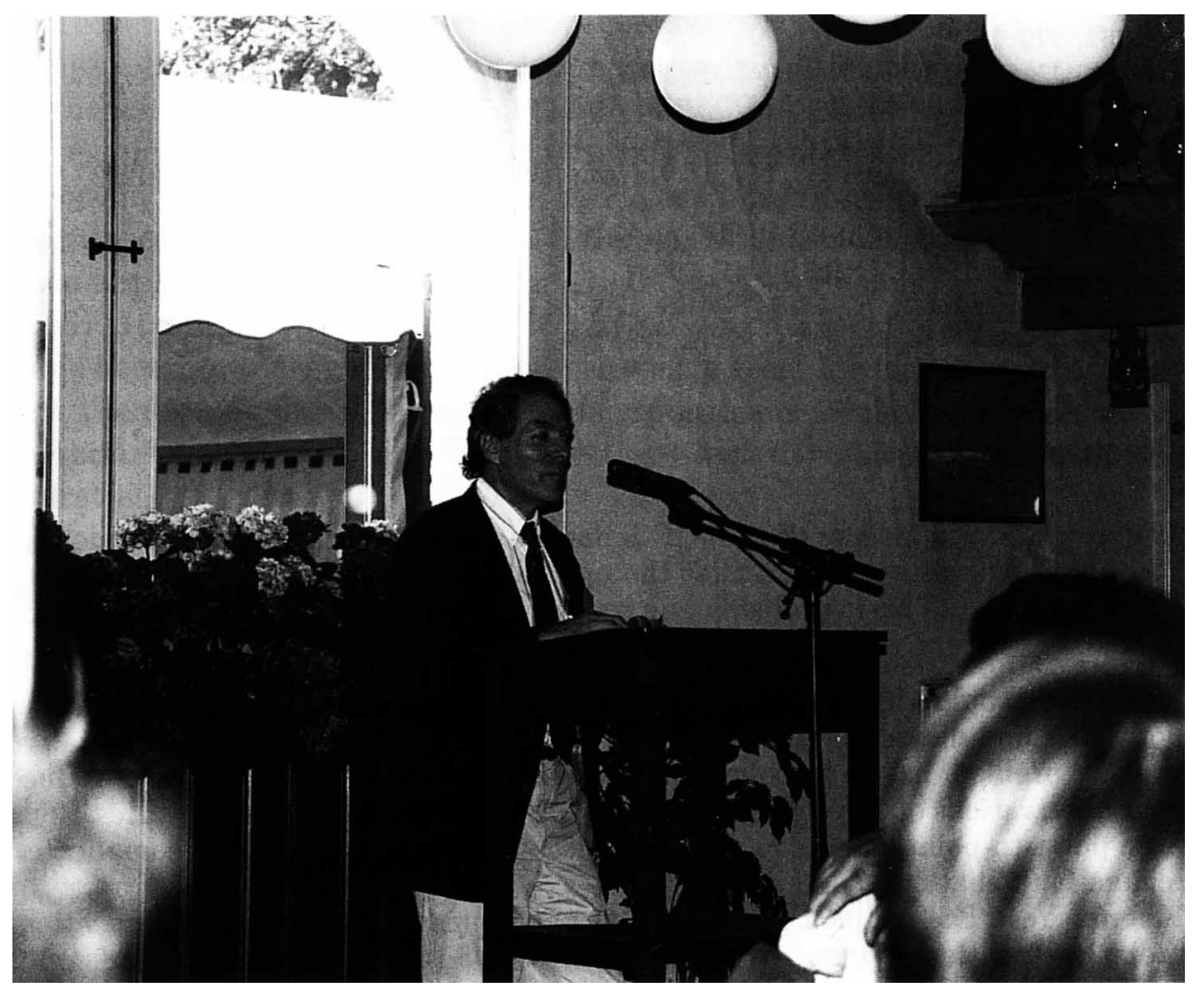

